\title{
A Commentary Article on: DNA Replication, Telomere Function and Cancer Action
}

\author{
Adnan Y Rojeab* \\ Electrical and Electronic Engineering Department, The London College, UCK, London, UK
}

Bio - magnetic and bioengineering are the applications of the electrical and magnetic signals on the living kind. It is, then, concerning with the living kind activity and other cooperation. In this commentary, it may be shown, generally, that in the spite of important of the enzymes, proteins, hormones and other biological factors to the whole processes of the biological actions in living life, but the electromagnetic signals are, possible, to be controlling the whole processes of the living kind activity.

The cloning process had accepted the challenge of electromagnetic signal in its result, and to yield the goat Dolly by the action of the magnetic field, [by applying a tiny electrical current], on dormant genes of a cell.

It is believed that, the main concerning matter of magnetic application on dormant genes is concerning, also, with that results arises from DNA replication, telomere function and that of cancer occurrence and cure treatments.

Also, now, it could be started with the research titled "The processes of DNA replication and the shortening of the telomere are influenced by the action of the magnetic field", where to say that, the effects are based on the electrical and magnetism action, as the fundamental phenomena, [electromagnetic], acting continuously on human and other kind living.

According to cloning and other applications, it can be shown that, magnetic phenomenon is, also, has its important action on DNA replication process and on the shortening of the telomere. Where, induction and remanat magnetisation are playing their great role. They are acting on the surrounding amino acid medium, [of the nucleus of the cell], by the magnetic field strength of each DNA strand, where the new [i.e. daughter] strand is created.

Accordingly, when the method of the DNA replication process is well and deeply explained, then it is believed that such an understanding is going to discover more about inherent operation, structure and activity of the process. Hence, clinically, it may be possible to improve some functions, as that of using a magnetic field.

Also, it can be said that, as the fundamental understanding to the process of DNA replication will lead to possible improvement to the performance of the DNA from the stage of original cell fertilization. This been shown on possible genetic orientation, as that the action been done by electromagnetic in the example of cloning process. A tiny magnetic field, from an electrical current, is applied to a cell. The magnetic field, now, operates as an anti - dormant factor on the genes of the chromosomes in the cell. It is to let all of the genes, in the cell, to be active instead of only, usually, some certain genes, which are belong to that certain tissue of the body. The magnetic action, on the cell, was as if it is forcing the whole cell to becoming just fertilized at once. For the DNA replication matter, each strand is applying by its magnetic field action.

As a matter of the DNA replication process, a shortening of the telomere is naturally occurred in the somatic cells. This process is performed according to the phenomenon that acting by remanant, [residual], magnetisation. Therefore, it is believed that, the magnetic field is the main action in the DNA replication.

Accordingly, it is believed that, wisely to be aimed to performed the process of shortening of the telomere, is by the action of the magnetic field. Where it is to change the number of base pairs, [which is the size of the telomere length]. Therefore, as it is shown, this application is, usually, applied during the process of the daughter strand creation, at DNA replication.

The action of the electromagnetic phenomenon is concerned with that of cloning operation process, and it is, [mainly], the matter of that of DNA replication. The result is the shortening of the telomere size.

Then, for the matter concerning with the telomere, DNA replication is leaving the somatic cells to have shortening of their telomere, while for the cancer cells the telomere is lengthening, [by the action of enzyme telomerase].

Hence, accordingly, there is suggestion that, during the DNA replication process, it is important to use the action of a magnetic field to apply on: the somatic cells, for a certain reason, and for those of cancer cells, for another reason.

Now, using the somatic cell, to be under a certain matter of improvement as well as to understand the reaction, by the applying the action of the magnetisation: the main process is to apply an action of a magnetic field, during DNA replication. The application is using variety of amounts and directions of a magnetic field strength, acting on the DNA molecule, during the replication process.

Hence, according to this aim, it is, mainly, to show whether, [or not], the magnetic action against DNA, is possibly arising any sort of changes. The changes are such as that of the manner of activities, deformation or of any other different structure in the shape. Accordingly, such a performance application will give a vast knowledge, as that for the deep understanding of the nature of DNA function and its process of replication.

Also, accordingly, it is important, in this manner, to show some comments on the function of the telomere:

First: it is believed that there are certain telomere characteristics, which are concerning with the structure and strength of the cell, as a

*Corresponding author: Dr. Adnan Y. Rojeab, Electrical and Electronic Engineering Department, The London College, UCK, London, U.K, Tel: +44 20 7243 4000; E-mail: rojeab@btinternet.com

Received: November 20, 2015; Accepted: November 26, 2015; Published December 15, 2015

Citation: Rojeab AY (2015) A Commentary Article on: DNA Replication, Telomere Function and Cancer Action. J Bioengineer \& Biomedical Sci 6: 174. doi:10.4172/2155- 9538.1000174

Copyright: () 2015 Rojeab AY. This is an open-access article distributed under the terms of the Creative Commons Attribution License, which permits unrestricted use, distribution, and reproduction in any medium, provided the original author and source are credited. 
whole. This is according with that pointing out for the understanding of the measuring matter of the level of the activities and mobility of the body as a whole. This may be explained as the following:

The shortening of the telomere of the DNA, is a continuing matter with frequent of the DNA replication, i.e. with the growth life of the kind living. Then, this means that it is an inversely relationship between the growth, oldness, of the kind living, and between that of the reduced, (loosing), the number of the base pairs, [i.e. shortening] of the telomere. The lower the number of the base pairs is the older, aged, of the kind living. This is to shown that,[at certain age of the life], as the kind living will be in the older [aged] situation, then it is the weaker in the general activity of the cell and that of the body, as a whole. This may show that, after a certain age: the shorter is of the telomere, it is the inhibiting, [weakening], of the ability and activity of the kind living. The importance of this characteristic is to point out to the aged matter of the cell which been affected by the function of the telomere. This can be shown, for an example, as that for an old and weighted, [great number of cells], person, is usually, having less activity than that of a young person of less, but reasonable, weight.

Second: comment on the critical length of the telomere, number of the base pairs:

Starting from the point: it is known that the critical number of the base pairs, [telomere length], is causing the DNA to be stopped for its replication process, until the cell dies. It is believed that cause of this occurrence is to save the genes, [of the DNA molecule], characteristics of that exact certain kind living. This matter can be discussed as the following: suppose that, the DNA is continued to be replicated after the stage of the critical length of the telomere, then the next DNA replication will cause some of the genes, which are near the telomere, to be lost. Then, this process will leave the DNA molecule, and then the cell, [as a whole], to be a totally different, in its genetic type, than that of its original one. This means that, the DNA molecule is going to be belong to some other kind of living, and not to the original one. Accordingly, the mechanism of the dying of the cell is right action to prevent any fundamental changes could be happed.

Now, it could be discussed what concerning with the cancer matter. It is believed that, a right thoughtful is to apply as the action of magnetism, [or electromagnetic action], in the aiming of the possibility to cure cancer.

Starting from the knowing fact that, the shortening of the telomere is the result of the natural process of DNA replication, [which is shown in the somatic cells].

Then, it is, believed, that for the possibility to cure the cancer, an action by using a process for the shortening of the telomere is an important aim. This can be performed by using the acting of the magnetic field.

Accordingly, a deliberate shortening to the telomere of the DNA, applied by an external magnetic action, could lead to change the operation, and then performance, of the cancer cells. This is a method of a treatment to be applied for the possibility to change the function, [fundamental activity], of the cancer cells, where, to lead them to behave in a manner as for those of ordinary somatic cells.

On the other hand, it should be realised that, it is, also, true, that the lengthening of the telomere is entirely important and fundamental process for some type of cells, in the kind living. It is the natural and right matter of creating and existing of the germ cells, [as sperms and ova], and, also, for other types of similar function, as those of stem cells.
It can be said that, the cancer is the matter of fully understanding to the behaviour of the DNA replication, and, also, to the origin of the cancer creation and the function of its mechanism. The aim of understanding may lead to advance stages to cure the cancer, when more studies are to be performed in some clinical stations.

In the cancer work, titled "Novel strategy of a method to cure the cancer", it is suggested that, the cancer is a mechanism aiming to eliminate the sever danger actions occurring against the vital genes in the cells. Then, the cancer is not a danger by itself, while, the dangerous matter are by some other operating traditional methods which are used in trying to cure it.

It is believed that, one of the suggestions is to apply a certain magnetic field strength to act accordingly on the cancerous cells. The aiming of this process is to reduce the number of the base pairs been added by the enzyme telomerase, to lengthening the telomere,[during DNA replication]. The magnetic action is, then, accounted, as an opposing process to the function of the enzyme telomerase. This is practically means that, as if a certain force is acting on the DNA to be replicated in a similar manner of that of the originally traditional somatic cells.

Hence, for the direct application to the cancer cells, the applied magnetic field should be arranged, [during the DNA replication], for a certain amount and direction, to be possibly acting against the enzyme telomerase. Therefore, it may show a shortening of the telomere instead of lengthening it, [for the similar characteristic of the somatic cell].

In other words, it is very important to apply a suitable method to be able to inhibit the action of the enzyme telomerase, where it is believed that, a magnetisation is an important application in this manner.

\section{A comment on existing of the magnetic field:}

It is, believed, that both the appearance and absent of the magnetic field are very important performance for the replication matter of the DNA, and then for the cell and the kind living as a whole.

It can be discussed this situation as that: The replication of DNA is associating with the unfold of the two strands. Where each strand will act by its own magnetic effect on the surrounding nucleus amino acid, to create the daughter strand. This process is very important, as it is the original and fundamental for the continuation of the exact type of gene characteristics of the same kind living. The important of the performance of this process is that, it is only working for a certain time, [replication time], and, also, for certain stage of the living cell function, [before die].

Also, it is fundamentally important to prevent, fully, the action and appearance of the magnetic field in the DNA molecule [as a whole entity]. This is because the continuation of the appearance of the magnetic effect in the DNA molecule, [as it is a whole entity], will show a continuance replication process without stopping. Hence, this occurrence will lead to that the number of chromosomes, in the cell, will be varied and in an increasing matter. As to suppose that, if it is happened, then, the cell situation will, obviously, show a great fundamental change to that of the original type of the kind living.

Accordingly, for the matter of the continuation of the life process of the certain kind living, both exposing and non- exposing the magnetic field action, at different stages of the DNA function, are of great important.

At for the final stage of this commentary, it is important to point 
Citation: Rojeab AY (2015) A Commentary Article on: DNA Replication, Telomere Function and Cancer Action. J Bioengineer \& Biomedical Sci 6: 174. doi:10.4172/2155- 9538.1000174

Page 3 of 3

out, again, for the requirement of some of clinical studies in the order to assess the level of the validating for the ideas been risen. Hence, it may be possible for the continuing application is to reach an advanced stage, as that for the matter of the improvement to the whole of DNA replication and cure the cancer. 